\title{
Pneumomediastinum after Orthognathic Surgery: Case Report and Review of the Literature*
}

\author{
Susanne Jung ${ }^{1 \#}$, Thomas Prien², Claudia Rudack ${ }^{3}$, Johannes Kleinheinz ${ }^{1}$ \\ ${ }^{1}$ Department of Cranio-Maxillofacial Surgery, University Hospital Muenster, Muenster, Germany \\ ${ }^{2}$ Department of Anaesthesiology, University Hospital Muenster, Muenster, Germany \\ ${ }^{3}$ Department of Otorhinolaryngology, University Hospital Muenster, Muenster, Germany \\ Email: \#Susanne.Jung@ukmuenster.de
}

Received February 5, 2013; revised March 9, 2013; accepted March 18, 2013

\begin{abstract}
Orthognathic surgery in general addresses young patients and aims to improve their bite function and to harmonize their facial aesthetics. Secure surgical standards and a defined post-surgical protocol of after-care are indispensable to reduce surgical as well as anaesthesiological risks in this area of complex elective surgery. The development of pneumomediastinum is a rare incident but threatens the patients' physical integrity. The case of a young healthy male who underwent Le Fort I osteotomy in combination with bilateral mandibular sagittal split osteotomy and postoperatively developed pneumomediastinum is presented, together with a discussion of the possible reasons for this rare complication of orthognathic surgery. The avoidance of life-threatening coincidences must be one main focus in the preparation and aftercare in elective orthognathic surgery.
\end{abstract}

Keywords: Pneumomediastinum; Pneumothorax; Mediastinal Emphysema; Orthognathic Surgery; Complication

\section{Introduction}

Orthognathic surgery knows a plethora of rare and unexpected complications: injuries to nearly all cranial nerves, cerebral complications including intracerebral bleeding, stroke or infections, i.e. brain abscesses and injuries to the extremities have been observed [1].

The manipulation in the upper aero digestive tract favors the occurrence of respiratory complications: the swelling of soft tissues, bleeding and the occurrence of mucous plugs obstructing the lower airways pave the way for the incidence of respiratory distress.

Pneumomediastinum is diagnosed, when the presence of free air in the mediastinum is pictured in the chest radiograph or the thoracic computer tomography. Secondary mediastinal emphysema results from a traumatic violation of the cervical or pulmonary tissues. From the exclusion of a causal trigger the diagnosis of spontaneous pneumomediastinum is made.

Spontaneous pneumomediastinum typically occurs under physical strain or asthma attack, situations that come

\footnotetext{
*Consent: Written informed consent was obtained from the patient for publication of this case report.

Authors' contributions: SJ and TP made substantial contributions to conception and design of this case report and drafted the manuscript. $\mathrm{CR}$ and $\mathrm{JK}$ were involved in the acquisition and interpretation of the data and in revising the article. All authors read and approved the final manuscript.

${ }^{\#}$ Corresponding author.
}

with an increased intrathoracic pressure. It affects generally healthy young men with a varying incidence between 1 of 800 to 1 of 42,000 patients [2]. The characteristic clinical course is generally benign.

Secondary pneumomediastinum is the result of a (blunt) traumatic injury of the mediastinal planes; in about $0 \%$ to $20 \%$ of the adult patients with thoracic trauma a pneumomediastinum occurs.

Due to the underlying pathology secondary mediastinal emphysema is often more complicated with a prolonged regeneration. The clinical features are unspecific and include acute chest pain, difficulties and hurting in swallowing, back ache, coughing and dyspnea [3].

We report a case of an 18-year-old male who suffered from a pneumomediastinum two days after orthognathic surgery.

\section{Case Report}

The 18-year-old healthy male, $64 \mathrm{~kg}, 180 \mathrm{~cm}$, presented for surgical correction of his skeletal class III and his pronounced mandibular prognathism after corresponding orthognathic preparation. His chief complaints were his severe malocclusion, impeded speech, and poor aesthetics. Clinical investigation revealed an anterior open bite, $6 \mathrm{~mm}$ reverse overjet and an inharmonic labial profile with protruding lower lip and incompetent lip closure.

The standard surgical planning included after ortho- 
dontic preparation a Le Fort I osteotomy with midfacial advancement, bilateral mandibular sagittal split osteotomy aiming for the correction of the lateral deviation and retrusion of the mandibula. Standard preoperative assessment contains laboratory diagnostics and the preclusion of infectious diseases or general health conditions associated with high anaesthesiological risks. The medical records and preoperative physical examination of the patient were entirely unremarkable.

The patient underwent the planned surgical intervention without surgical or anaesthesiological difficulties or complications. The operation lasted 150 minutes and terminated with the intermaxillary fixation according to the standard procedure to stabilize the skeletal shift for the coming 3 days. The removal of the nasoendotracheal tube immediately after surgery with intermaxillary fixation in place, the postanaesthesia recovery phase and the 24-hours observation interval on the intermediate care unit were uneventful.

24 hours after surgery, the patient was transferred to the peripheral ward. During the second postoperative night, approximately 26 hours postoperatively, the patient suddenly developed respiratory distress without any physical effort and complained from retrosternal chest pain, arousing him from sleep. The peripheral oxygen saturation was $92 \%$ without oxygen supply; the heart rate was $126 \mathrm{bpm}$ and the blood pressure $155 / 80 \mathrm{mmHg}$. Clinical investigation presented no further pathologies but for peripheral cyanosis; the intermaxillary fixation was removed immediately. The intraoral findings were regular: no bleeding or intensive secretion could be detected. The aspiration of parts of the patient's braces was excluded.

The initial diagnostic intervention included an ECG, the laboratory investigation of heart specific enzymes, and a CT-scan of the thorax to exclude pulmonary embolism or myocardial infarction. A vascular obliteration could be excluded but the CT scan revealed a massive pneumomediastinum with emphysema retrosternally from the diaphragm beyond the aortal arch up to the retropharyngeal area (Figure 1). The expanse of the airy pericardial hemline was $3 \mathrm{~mm}$ (Figure 2).

To prevent further air trapping via undetected tracheal or pharyngeal tissue laceration, pan-endoscopy, esophagoscopy and bronchoscopy were performed in general anesthesia, to recognize and eventually close a suspected penetrating injury. The fibreoptically guided reintubation of the awake patient 35 hours after orthognathic surgery was complicated by a pronounced swelling of the facial soft tissues and difficult due to poor compliance of the patient. During endoscopy a small hematoma in the left lateral pharyngeal wall was identified. Additionally, two small lacerations, one in the right nasal cavity and one in the posterior pharyngopalatine arch were detected and

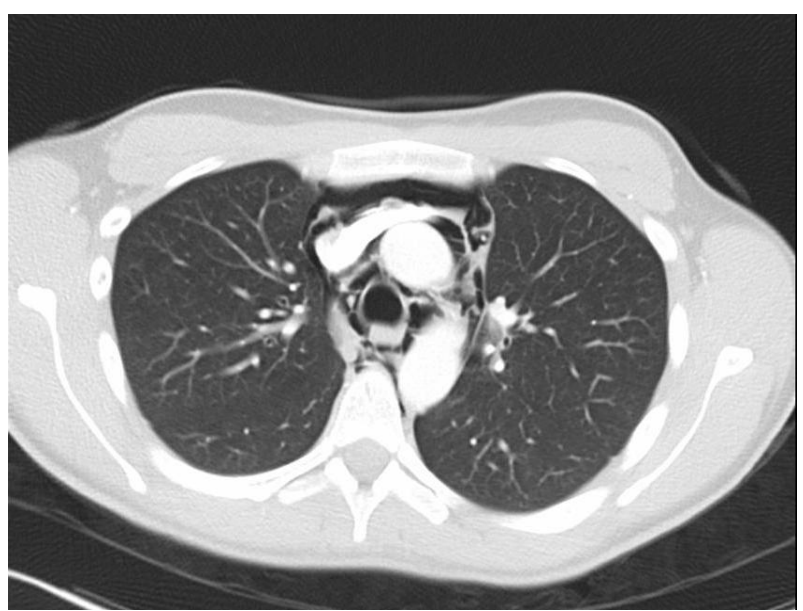

Figure 1. Thoracic CT scan: Massive pneumomediastinum with emphysema retrosternally from the diaphragm up to the retropharyngeal area.

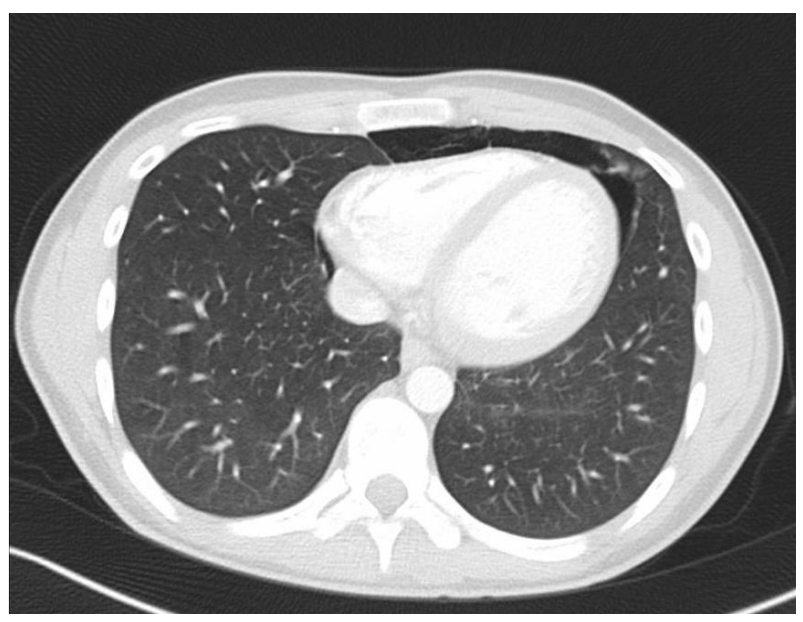

Figure 2. Thoracic CT scan: Expanse of the airy pericardial hemline $3 \mathrm{~mm}$.

sutured. Despite a thorough inspection, a relevant penetration of the cervical fascia as a possible site for air entry into the retropharyngeal tissues was not found.

Consequently, the causal relation of the detected small wounds and the massive pneumomediastinum was neither proven nor excluded by panendoscopy. To avoid further physical distress and to allow for possible closure of an undetected supraglottic site of air entry, the removal of the orotracheal tube was delayed for two days. The patient was transferred to the intensive care unit for surveillance and hemodynamic and cardiac monitoring. Under continuous ventilation there was no progression of the mediastinal emphysema, but a successive resorption of the pneumomediastinum could be illustrated radiologically, rendering a subglottic site of air entry implausible.

Two days later, the patient was weaned off ventilation, was extubated and his oxygen saturation remained stable 
over 97\%. With clinical and laboratory signs of beginning pulmonary infection, an antibiotic therapy with piperacillin/ tazobactam was initiated and maintained for 5 days, when signs of infection had resolved.

Follow-up thoracic CT scans documented the continually proceeding resorption of the intramediastinal air (Figure 3(a) and (b)).

The patients' recovery was uneventful, the result of the corrective jaw surgery was untouched, and there are no long-term sequelae to be expected. The duration of inpatient care until discharge was 12 days.

The postoperative ambulant assessment and physical examination four weeks after surgery was unremarkable. The patient reported no physical impairment or other complications secondary to the postoperative pneumomediastinum.

\section{Discussion}

Orthognathic surgery includes corrections of the somatognatheous system that are generally performed electively. In most cases the patients concerned are young,

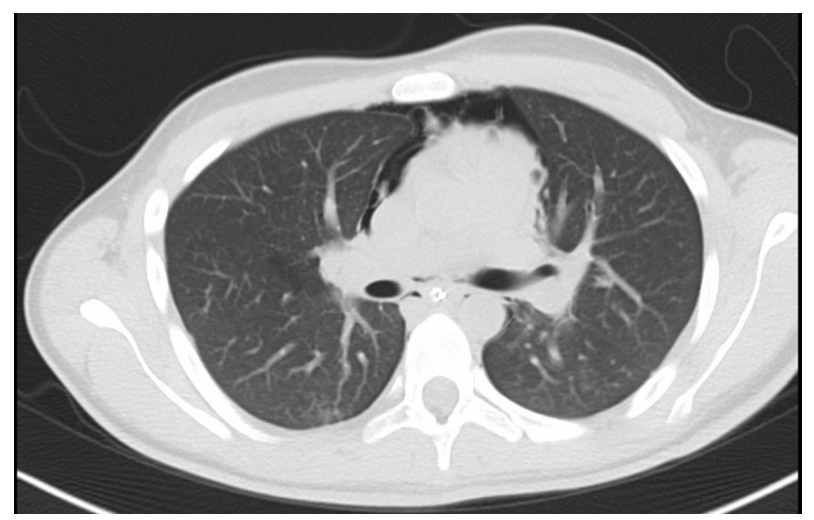

(a)

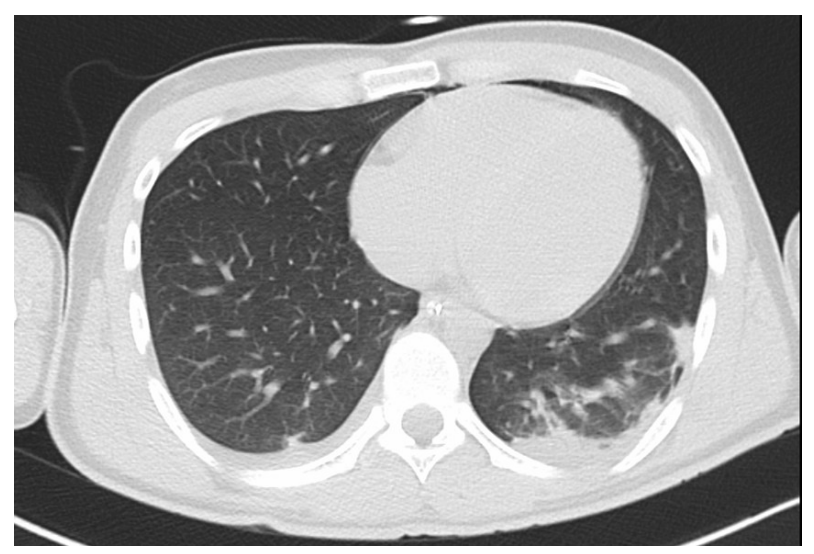

(b)

Figure 3. Follow-up thoracic CT scan: (a) Proceeding resorption of the intramediastinal air on day 2 after surgery; (b) Continualresorption of the intramediastinal air on day 3. healthy and their medical history is insignificant.

Most common complications in orthognathic surgery are bleeding, nerve damage or relapse. The development of a pneumomediastinum or a pneumothorax after orthognathic surgery is a rare but possibly life threatening complication that has been described in 6 cases to date [4-6].

There are different pathomechanisms and pathways by which air or gas may gain access to the mediastinum. Anatomically, there is a continuum between the paraand retropharyngeal spaces, the visceral space of the neck, the mediastinum, the peribronchial, and the retroperitoneal space, allowing gas in any of these spaces to move to the other sites alongside pressure gradients. Pressures within the intrathoracic structures are always lower than alveolar pressures, regardless whether the patient is ventilated or spontaneously breathing, forcing air through alveolar, bronchial, or tracheal leaks of any origin [7]. The subatmospheric pressure within the thoracic cavity under spontaneous breathing may also-to varying degrees - be transmitted cephalad to the retropharyngeal space, thereby allowing air to enter here through mucosal tears. Otherwise, emphysematous complications in dentistry and maxillofacial surgery without application of positive pressure devices could not be explained [8].

Therefore, the combination of retropharyngeal and mediastinal emphysema may either be due to an air leak at the subglottic level with air migrating cephalad or, less often, to supraglottic injury with air migrating caudally.

In the case presented here, emphysema became clinically apparent approximately 36 hours after surgery. Two small mucosal lacerations, either due to nasal intubation, placement of a gastric tube, or orthognathic surgery were detected upon endoscopic inspection of the upper airways whereas tracheal injury definitely could be ruled out. Emphysema was regredient with reintubation and mechanical positive pressure ventilation. Thus, a subglottic site of air entry appears improbable, rather, the supraglottic mucosal injury with slow but steady air entry into the retropharyngeal tissues during spontaneous brea thing, migrating caudally into the mediastinum, most likely was the culprit.

The recommendations as to the adequate therapy of spontaneous and traumatic mediastinal emphysema are geared to the manifestation of the symptoms and the site of air entry. First therapeutic step in traumatic cases is the identification and reliable closure of the defect. Subsequently, in mild cases with sparse emphysema, symptomatic therapy, the avoidance of positive pressure airway and cardiac and hemodynamic monitoring on an intensive or intermediate care unit are appropriate. The supportive appliance of oxygen helps to ensure stable oxygen saturation and furthermore precipitates the re- 
sorption of the mediastinal or pleural air.

Decisive clinical parameters for the induction of further therapeutic intervention are blood pressure and heart rate: acutely developing hypotension and tachycardia may point to to a reduced cardiac output caused by tension pneumomediastinum or-thorax. When (tension-) pneumothorax is diagnosed the appliance of tube thoracostomy is indicated to release the pleural pressure and to allow for re-expansion of the lung. In contrast to the placement of a thoracical drain, the incision to drain a pneumomediastinum is located on the cranial end of the sternum. The re-expansion of the lung and the resorption of the emphysema together with the vital parameters have to be monitored regularly.

With special regard to maxillofacial surgery, the rigid intermaxillary fixation with its allegedly concomitant effects as secret-retention or impaired swallowing, as a possible trigger for the development of a spontaneous pneumomediastinum is discussed. We regard the rigid intermaxillary fixation for three days after surgery as prerequisite to grant an optimal orthognathic outcome; an omission to prevent respiratory distress as it is recommended in literature is not necessary when close postoperative surveillance and protocols for airway management are integrated. Such procedures include the postoperative observation on an intermediate care unit and a standardized protocol of aftercare including among others wire shears that are continually kept on hand [9].

The crucial question remains: How to avoid this severe complication after orthognathic surgery?

The first area of interest is the anaesthesiogical treatment with gentle handling of the airways and the pharyngeal soft tissues. When the patients wake from anaesthesia it is most important to reduce any cause of intrathoracic pressure as coughing or regurgitation.

The surgical intervention has to be performed with utmost care, not only for the teeth and skeletal structures but also for the soft tissues of the soft palate and the pharyngeal walls.

The risk for the development of mediastinal emphysema can be reduced by respecting the requirements of the structural conditions but cannot be fully excluded, so the surgeons and anaesthetists have to pay attention to the relevant symptoms.

Gentle removal of the nasotracheal tube, thorough drainage of oral and nasal cavity and suction of swallowed blood via nasogastric tube, avoidance of tracheal swelling, application of detumescing nose drops, efficient habitual mouth care and regular oral suction during intermaxillary fixation represent the basis for stable respiratory conditions, timely wound healing and a short period of hospital stay.

The avoidance of life-threatening coincidences must be one main focus in the preparation and aftercare in elective orthognathic surgery. The preponderance of young and fit patients must not dim the wakefulness regarding the imminent respiratory risks of oral and maxillofacial operations.

\section{REFERENCES}

[1] B. J. Steel and M. R. Cope, "Unusual and Rare Complications of Orthognathic Surgery: A Literature Review," Journal of Oral and Maxillofacial Surgery, Vol. 70, No. 7, 2011, pp. 1678-1691.

[2] M. Chalumeau, L. Le Clainche, N. Sayeg, N. Sannier, J. L. Michel, R. Marianowski, P. Jouvet, P. Scheinmann and J. de Blic, "Spontaneous Pneumomediastinum in Children,” Pediatric Pulmonology, Vol. 31, No. 1, 2001, pp. 67-75.

[3] S. D. Pryor and L. K. Lee, "Clinical Outcomes and Diagnostic Imaging of Pediatric Patients with Pneumomediastinum Secondary to Blunt Trauma to the Chest," The Journal of Trauma, Vol. 71, No. 4, 2011, pp. 904-908.

[4] N. A. Chebel, D. Ziade and R. Achkouty, "Bilateral Pneumothorax and Pneumomediastinum after Treatment with Continuous Positive Airway Pressure after Orthognathic Surgery," British Journal of Oral and Maxillofacial Surgery, Vol. 48, No. 4, 2010, pp. e14-e15.

[5] D. B. Edwards, R. B. Scheffer and I. Jackler, "Postoperative Pneumomediastinum and Pneumothorax Following Orthognathic Surgery,” Journal of Oral and Maxillofacial Surgery, Vol. 44, No. 2, 1986, pp. 137-141.

[6] J. F. Piecuch and R. A. West, "Spontaneous Pneumomediastinum Associated with Orthognathic Surgery. A Case Report," Oral Surgery, Oral Medicine and Oral Pathology, Vol. 48, No. 6, 1979, pp. 506-508.

[7] R. J. Maunder, D. J. Pierson and L. D. Hudson, "Subcutaneous and Mediastinal Emphysema. Pathophysiology, Diagnosis and Management," Archives of Internal Medicine, Vol. 144, No. 7, 1984, pp. 1447-1453. doi:10.1001/archinte.1984.00350190143024

[8] S. N. Heyman and I. Babayof, "Emphysematous Complications in Dentistry, 1960-1993: An Illustrative Case and Review of the Literature,” Quintessence International, Vol. 26, No. 8, 1995, pp. 535-543.

[9] T. Kim, J. Y. Kim, Y. C. Woo, S. G. Park, C. W. Baek and H. Kang, "Pneumomediastinum and Pneumothorax after Orthognathic Surgery: A Case Report,” Korean Journal of Anesthesiology, Vol. 59, 2010, pp. S242-S245. doi:10.4097/kjae.2010.59.S.S242 